\title{
A Unusual presentation of Bordetella pertussis
}

\begin{abstract}
Bordetella pertussis is known to be a highly contagious organism leading to variable clinical manifestations especially in children. Severe pulmonary hypertension, apneas, convulsions and failure to thrive have been reported as symptoms associated with the infection however edema associated with $B$. pertussis illness is poorly reported in literature. In this report the clinical association between $B$. pertussis and generalized edema in infants is highlighted. By excluding all other causes, the infant's edema was thought to be due to a capillary leak resulting from inflammation mediated by toxins produced by $B$. pertussis.
\end{abstract}

Volume 7 Issue 3 - 2017

\section{Ziad Chebel, Nadine Jawad, Yolla Nassif,} Robert Sacy, Dany Al Hamod

Department of Pediatrics, Saint George Hospital University

Medical Center, University of balamand, Lebanon

Correspondence: Dany Al Hamod, Department of Pediatrics, Saint George University Hospital, University of Balamand, Beirut, Lebanon,Tel 961-368-3209, Email dany_alhamod@hotmail.com

Received: October 01, 2017 | Published: October 23, 2017
Abbreviations: AC, adenylyl cyclase; EF, edema factor; Ptx, pertussis toxin; RAAS, renin-angiotensin-aldosterone system

\section{Introduction}

Bordetella pertussis, the gram-negative bacterial pathogen causing Pertussis, is known to be a highly contagious organism leading to variable clinical manifestations especially in children.

In 2013, an estimated mortality of approximately 400 per million live births due to pertussis was reported in the first year of life. ${ }^{1}$ While unvaccinated children are affected with the most severe clinical manifestations of the infection, vaccinated children can still show characteristic symptoms. The clinical spectrum of $B$. pertussis infection ranges from a mild illness to severe illness leading to death. ${ }^{2}$ Severe pulmonary hypertension, apneas, convulsions and failure to thrive have been reported as symptoms associated with the infection, ${ }^{3,4}$ however edema associated with $B$. pertussis illness is poorly reported in literature. In this report the clinical association between $B$. pertussis and generalized edema in infants is highlighted.

\section{Case report}

A 2 month old baby boy was admitted to our hospital for the management of severe respiratory distress and generalized edema. He was delivered vaginally at term to a G3P3A0 mother, who underwent an uneventful pregnancy, the baby was healthy and doing well until 12 days prior to his presentation at our hospital; the patient was noted by his mother to have a recurrent wet cough mostly postprandial with no other apparent signs and symptoms and so was diagnosed with reflux and given symptomatic treatment. Two days later he developed a low grade fever and was started by his physician on Cefpodoxime. Patient did not improve over the next $48 \mathrm{hrs}$, the fever started reaching 40 degrees Celsius and the baby was having paroxysmal cough so was admitted for treatment of suspected Pertussis infection by IV clarithromycin.

During his stay the patient was clinically deteriorating, he soon developed generalized pitting edema $2+$ and a tonic clonic generalized seizure that lasted a few minutes for which intrarectal diazepam was administered and the patient was immediately transferred to an intensive care unit for better management and observation. Upon admission, physical examination demonstrated an afebrile, edematous baby weighing $8400 \mathrm{~g}$ with a respiratory rate of 65 breaths/minute with slight inter and subcostal retractions, nasal flaring and a persistent paroxysmal cough oxygen saturation was $94 \%$ on room air, with a heart rate of $190 \mathrm{bpm}$ and a $\mathrm{BP}$ of $130 / 40 \mathrm{mmHg}$. Auscultation of the lungs disclosed diffuse rales and rhonchi. Heart sounds were normal no murmurs detected and normal pulses. Capillary refill was 3 seconds and hepatomegaly was noted on physical examination. The edema was generalized pitting, most evident on the extremities, eyelids, head, abdomen and sacrum.

Upon arrival a CT brain was done which was normal then a lumbar puncture followed which showed normal cell count, negative culture and respiratory and bacterial. The patient was having desaturations and worsening of his respiratory status so he was intubated/ventilated, sedated (on Midazolam and Fentanyl) and placed on CMV mode. A central line was inserted for better IV access. Echocardiography done showed good contractility with no abnormalities seen, the patient also developed tachycardia reaching $250 \mathrm{bpm}$ so an ECG done showed sinus rhythm and repeat echocardiography was unchanged.

Laboratory examination showed CBC: WBC $44.10^{\wedge} 3 / \mathrm{mm}$, neutrophils $42 \%$, lymphocytes $53 \%$ hgb $9 \mathrm{~g} / \mathrm{dl}$, hct $27 \%$ plts $244.10^{\wedge} 3 /$ mm MCV 94fl, PT INR 1.68, PTT 37sec. Electrolytes (meq/L): sodium 124 , potassium 4.4 chloride 103 , bicarbonate 28 , urea $19 \mathrm{mg} / \mathrm{dl}$ creatinine $0.21 \mathrm{mg} / \mathrm{dl}$, protein $4.1 \mathrm{~g} / \mathrm{dl}$ albumin $3.6 \mathrm{~g} / \mathrm{dl}$, calcium $8.7 \mathrm{mg} /$ $\mathrm{dl}$, phosphorus $3.6 \mathrm{mg} / \mathrm{dl}$, mag $1.6 \mathrm{mg} / \mathrm{dl}$, uric acid $0.9 \mathrm{mg} / \mathrm{dl}$ glucose $49 \mathrm{mg} / \mathrm{dl}$, procalcitonin $71 \mathrm{ng} / \mathrm{ml}$, CRP $0.08 \mathrm{mg} / \mathrm{dl}$ liver enzymes were all normal, TG and cholesterol were normal. Chest Xray showed a normal sized heart with cardiac index of 0.55 , with congested blunted costophrenic angles and diffuse infiltrates so patient was started empirically on Meropenem and Teicoplanin. Respiratory viral panel was sent and was negative. Blood cultures and urine cultures also sent came back negative.

The patient was initially placed on lasix $0.5 \mathrm{mg} / \mathrm{kg} \mathrm{q} 6 \mathrm{~h}$ in addition to spironolactone $8 \mathrm{mg}$ qd and fluid restriction was set at a total fluid input of $60 \mathrm{cc} / \mathrm{kg} /$ day. However, the patients edema had not improved so a repeat xray was done CI was 0.6. Lasix continuous was started at $0.1 \mathrm{mg} / \mathrm{kg} / \mathrm{hr}$ and urine output was monitored.The patient started urinating $7 \mathrm{cc} / \mathrm{kg} / \mathrm{hr}$ so spironolactone was halted and lasix continued. Workup was initiated for evaluation of the edema; nephrologic causes needed to be ruled out like nephrotic syndrome TG/cholesterol, albumin, urine spot all normal.

Renin and aldosterone levels were mildly elevated, and cortisol level was normal. Thyroid workup was normal. Immune workup was ordered including IgG, IgM, IgA, IgG subclasses, C3,C4 and flow cytometry and turned out normal. CF mutations ordered was also normal. Abdominal ultrasound done showed a fatty enlarged liver 
with thickened gallbladder wall no fluids in the abdomen complete liver panel came back normal.

By this point the patient had been intubated for 12 days, despite the treatment the patient was still very edematous and nonextubateable, with frequent mucous plugs and desaturations requiring frequent suctioning and lavage. However, the inflammatory markers started to trend down, so lasix dose was adjusted to $0.2 \mathrm{mg} / \mathrm{kg} / \mathrm{hr}$ and the infectious disease team opted for a Bronchoalveolar lavage which displayed atelectasis of the right lung after the right main bronchus and thick mucoid secretions which were sent for culture, cell count and bacterial and viral studies. Respiratory viral panel repeated on the BAL came back positive for bortedella pertussis so Clarithromycin was added and as soon as the withstanding pancultures came back negative meropenem and targocid were halted.

The patient started to lose the water retention gradually dropping from the initial $8.4 \mathrm{~kg}$ to a whooping $6.8 \mathrm{~kg}$, clinically he was requiring less and less parameters on the respirator and was successfully extubated on day 12 of his ICU stay. His lasix dose was tapered slowly and the patient was feeding the second day after extubation. On the third day after his extubation the patient was transferred to the floor.

\section{Discussion}

Respiratory distress, apneas, hyponatremia, convulsions and edema were the clinical manifestations of $B$. pertussis in our infant. The history of cough, apneas and respiratory distress caused by $B$. pertussis infection during the catarrhal and paroxysmal phase showed a classical presentation at first. Although treatment was started in the paroxysmal phase, our patient did not improve and required intubation.

In a 2007 Cochrane systematic review about the use of antibiotics for whooping cough, Altunaiji et al. ${ }^{5}$ concluded that although antibiotic therapy was effective in the elimination of B. pertussis, it did not affect the subsequent clinical course of the illness. ${ }^{5}$ The reason behind this conclusion is explained by the fact that complications of pertussis are mainly caused by the toxins produced by $B$. pertussis which continue to be present after the eradication of the microorganism. ${ }^{3,6}$ Ciliated epithelial cells of lung tissues damaged by these toxins are thought to be the cause behind the paroxysmal cough of pertussis. ${ }^{7}$

Bronchopneumonia can occur leading to most fatal cases; this may be due to primary $B$. pertussis infection itself or to secondary infection with other respiratory microorganism. ${ }^{4}$ The initial stages of infection entail congestion and infiltration of the mucosa by lymphocytes and polymorphonuclear leukocytes. The lumens of the bronchi contain inflammatory debris.

In many reported cases, postmortem data show extensive hemorrhage in the lungs. ${ }^{8}$ Pulmonary edema and focal hemorrhages were also reported with diffuse infiltration by macrophages and inflammatory cells, associated with extensive necrotizing bronchopneumonia and thromboemboli. Widespread mucus plugs and extensive mucosal damage were previously reported ${ }^{9}$ which was also noted in our case.

Edema have not been previously described as the predominant clinical feature or complication of pertussis in infants before. One reported case found in literature described a similar presentation with a neonate who developed severe edema associated with neonatal $B$. pertussis infection. ${ }^{10}$

At first, the goal was to eliminate all the known causes of edema in infants: because of the combination of respiratory distress, hyponatremia and severity of the respiratory infection, cystic fibrosis was suspected. Nevertheless, CF mutations were ordered and turned out normal. Furthermore, there were no signs of renal sodium loss or a syndrome of inappropriate $\mathrm{ADH}$ secretion explaining the hyponatremia and edema. Cardiac evaluation and ultrasound of the abdomen did not reveal any abnormality that could have explained the edema. Edema associated with congenital causes like Noonan syndrome was not likely to start after the age of 2 months.

Once all the known causes of edema in infants were excluded, the $B$. pertussis toxins were thought to be the trigger of this presentation. B. pertussis produces several toxins, the adenylyl cyclase (AC) toxins edema factor (EF) and CyaA weaken the immune responses and facilitate bacterial proliferation. ${ }^{11}$ Interestingly, the toxin produced by Bacillus anthracis, the adenylyl cyclase toxin called edema toxin, falls in the same family of the toxin produced by $B$. pertussis. ${ }^{12}$ Severe edema is one of the most recognized clinical symptom of a Bacillus infection that is caused by an increase in the vascular permeability following a release of inflammatory mediators like histamine, prostanoids, and neurokinins. ${ }^{13}$ Sakuma S, et al. ${ }^{14}$ noted that Pertussis toxin (Ptx), the most known toxin, alters the vascular permeability of vessels outside the lung. ${ }^{14}$ Pertussis toxin (Ptx) also causes lung edema by activating cAMP and changing concentrations of protein kinase $\mathrm{C}$ and manganese superoxide dismutase. ${ }^{15,16}$

Capillary leak resulting from inflammation mediated by toxins may have caused the edema formation in our patient. The leak elicited extravasations of water and sodium into the interstitium resulting in diminished effective blood volume leading to the activation of the renin-angiotensin-aldosterone system (RAAS) causing retention of sodium and water.

\section{Conclusion}

Edema was not previously described as a main symptom of Bordetella pertussis infection in infants. By excluding all other causes of edema in our case, it was thought to be due to the capillary leak resulting from inflammation mediated by toxins produced by $B$. pertussis. Further in vitro and in vivo studies are recommended to establish a clear mechanism explaining the damage resulting from pertussis toxins to the capillary wall.

\section{Acknowledgments}

None.

\section{Conflicts of interest}

The authors declare no conflicts of interest.

\section{References}

1. Liu L, Oza S, Hogan D, et al. Global, regional, and national causes of child mortality in 2000-13, with projections to inform post-2015 priorities:an updated systematic analysis. Lancet. 2015;385:430-440.

2. Cherry JD. The history of pertussis (whooping cough); 1906-2015:facts, myths, and misconceptions. Current Epidemiology Reports. 2015;2(2):120-130.

3. Mattoo S, Cherry JD. Molecular pathogenesis, epidemiology, and clinical manifestations of respiratory infections due to Bordetella pertussis and other Bordetella subspecies. Clin Microbiol Rev. 2005;18(2):326-382.

4. Cherry JD, Heininger U. Pertussis and other Bordetella infections. In: Feigin RD, et al. editors. Textbook of pediatric infectious diseases, 5th edn), The W. B. Saunders Co, Philadelphia, USA. 2004. p. 1588-1608.

5. Altunaiji S, Kukuruzovic R, Curtis N, et al. Antibiotics for whooping cough (pertussis). Cochrane Database Syst Rev. 2007;18(3):CD004404. 
6. Hewlett EL. Pertussis:current concepts of pathogenesis and prevention. Pediatr Infect Dis J. 1997;16(4):S78-S84.

7. Cherry JD, Brunell PA, Golden GS, et al. Report of the task force on pertussis and pertussis immunization-1988. Pediatrics. 1988;81(6):939.

8. Christie CD, Baltimore RS. Pertussis in neonates. Am J Dis Child. 1989;143(10):1199-1202.

9. Smith C, Vyas H. Early infantile pertussis; increasingly prevalent and potentially fatal. Eur J Pediatr Dec. 2000;159(12):898-900.

10. Meine Jansen C, Miedema C. Edema as a new predominant symptom of Bordetella pertussis infection in a newborn. Eur $J$ Pediatr. 2009;168(12):1543-1545.

11. Göttle M, Dove S, Kees F, et al. Cytidylyl and uridylyl cyclase activity of bacillus anthracis edema factor and Bordetella pertussis CyaA Biochemistry. 2010;49(26):5494-5503.
12. Ahuja N, Kumar P, Bhatnaqar R. The adenylate cyclases toxins. Crit Rev Microbiol. 2004;30(3):187-196.

13. Tessier J, Green C, Padgett D, et al. Contributions of histamine, prostanoids, and neurokinins to edema elicited by edema from bacillus anthracis. Infect Immunol. 2007;75(4):1895-1903.

14. Sakuma S, Imagawa Y, Tokunaga E, et al. Increase in intradermal vascular permeability caused by pertussis toxin from Bordetella pertussis. Microbiol immunol. 1987;31(6):531-539.

15. Garcia JG, Wang P, Schaphorst KL, et al. Critical involvement of p38 MAP kinase in pertussis toxin-induced cytoskeletal reorganization and lung permeability. FASEB J. 2002;16(9):1064-1076.

16. Tsan MF, Cao X, White JE, et al. Pertussis toxin-induced lungedema. Role of manganese superoxide dismutase and protein kinase C. $\mathrm{Am} J$ Respir Cell Mol Biol. 1999;20(3):465-473. 